\title{
Enduring Critical Period Plasticity Visualized by Transcranial Flavoprotein Imaging in Mouse Primary Visual Cortex
}

\author{
Manavu Tohmi, Hiroki Kitaura, Seiji Komagata, Masaharu Kudoh, and Katsuei Shibuki \\ Department of Neurophysiology, Brain Research Institute, Niigata University, Niigata 951-8585, Japan
}

\begin{abstract}
Experience-dependent plasticity in the visual cortex was investigated using transcranial flavoprotein fluorescence imaging in mice anesthetized with urethane. On- and off-responses in the primary visual cortex were elicited by visual stimuli. Fluorescence responses and field potentials elicited by grating patterns decreased similarly as contrasts of visual stimuli were reduced. Fluorescence responses also decreased as spatial frequency of grating stimuli increased. Compared with intrinsic signal imaging in the same mice, fluorescence imaging showed faster responses with $\sim 10$ times larger signal changes. Retinotopic maps in the primary visual cortex and area LM were constructed using fluorescence imaging. After monocular deprivation (MD) of $4 \mathrm{~d}$ starting from postnatal day 28 (P28), deprived eye responses were suppressed compared with nondeprived eye responses in the binocular zone but not in the monocular zone. Imaging faithfully recapitulated a critical period for plasticity with maximal effects of MD observed around P28 and not in adulthood even under urethane anesthesia. Visual responses were compared before and after MD in the same mice, in which the skull was covered with clear acrylic dental resin. Deprived eye responses decreased after MD, whereas nondeprived eye responses increased. Effects of MD during a critical period were tested 2 weeks after reopening of the deprived eye. Significant ocular dominance plasticity was observed in responses elicited by moving grating patterns, but no long-lasting effect was found in visual responses elicited by light-emitting diode light stimuli. The present results indicate that transcranial flavoprotein fluorescence imaging is a powerful tool for investigating experiencedependent plasticity in the mouse visual cortex.
\end{abstract}

Key words: visual cortex; experience-dependent plasticity; flavoproteins; fluorescence; optical imaging; mouse

\section{Introduction}

Monocular deprivation (MD) produces experience-dependent plasticity in the visual cortex (Wiesel and Hubel, 1965; Hubel and Wiesel, 1970; Hubel et al., 1977; LeVay et al., 1980). This plasticity has been studied extensively in mice suitable for genetic engineering using electrophysiological recordings (Dräger, 1978; Gordon and Stryker, 1996), intrinsic signal imaging (Antonini et al., 1999; Cang et al., 2005a; Hofer et al., 2006), and histological analysis (Antonini et al., 1999; Tagawa et al., 2005). Electrophysiological recordings have merit in the quantitative analysis of neural activities, but a disadvantage is sampling bias. Intrinsic signal imaging is powerful for constructing cortical maps (Grinvald et al., 1986; Frostig et al., 1990; Kalatsky and Stryker, 2003), but the magnitude of neural activities might not be faithfully recorded by this method (Nemoto et al., 2004; Sheth et al., 2004; Zhan et al., 2005).

Endogenous fluorescence derived from NADH (reduced form of nicotinamide adenine dinucleotide) or flavoproteins has been used for measuring activity-dependent changes in brain metab-

Received April 17, 2006; revised 0ct. 4, 2006; accepted 0ct. 5, 2006.

This work was supported by grants from the Japanese Government. We thank Y. Tamura, N. Taga, and S. Maruyama for technical assistance.

Correspondence should be addressed to Dr. Katsuei Shibuki, Department of Neurophysiology, Brain Research Institute, Niigata University, 1 Asahi-machi, Niigata 951-8585, Japan. E-mail: shibuki@bri.niigata-u.ac.jp. DOI:10.1523/JNEUROSCI.1643-06.2006

Copyright $\odot 2006$ Society for Neuroscience $\quad$ 0270-6474/06/2611775-11\$15.00/0 olism (Chance et al., 1962; Shuttleworth et al., 2003). Flavoprotein fluorescence has been used for functional brain imaging in vivo (Shibuki et al., 2003; Coutinho et al., 2004; Reinert et al., 2004; Weber et al., 2004). Signal changes $\left(\Delta F / F_{0}\right)$ in flavoprotein fluorescence imaging are $\sim 10$ times larger than those in intrinsic signal imaging (Takahashi et al., 2006). The high spatial resolution of this technique has been demonstrated by studies in which localized activities along cerebellar parallel fiber bundles were visualized (Coutinho et al., 2004; Reinert et al., 2004). The stability of flavoprotein signaling is suitable for investigating activitydependent plasticity (Murakami et al., 2004) and learninginduced plasticity (Shibuki et al., 2006b) in the rat somatosensory cortex. This technique is especially useful for visualizing mouse cortical activities, because the skulls of mice are sufficiently transparent to allow transcranial imaging (Shibuki et al., 2006a). Transcranial flavoprotein fluorescence imaging has been used for the visualization of experience-dependent plasticity in the auditory cortex (Takahashi et al., 2006), the somatosensory cortex (Kitaura et al., 2005), and cortical epileptic foci in mice (Takao et al., 2006).

We investigated experience-dependent plasticity after MD in the mouse visual cortex using transcranial flavoprotein fluorescence imaging. Studies using field potential recordings have reported a decrease in deprived eye responses after MD in young mice and an increase in nondeprived eye responses in adult mice 
(Sawtell et al., 2003; Frenkel and Bear, 2004). In the present study, we confirmed that deprived eye responses decreased and nondeprived eye responses increased after MD in young mice. Longlasting plasticity induced by MD during a critical period has been demonstrated in adult animals (Wiesel and Hubel, 1965; Hubel and Wiesel, 1970; Hubel et al., 1977; LeVay et al., 1980). However, the presence of such long-lasting plasticity is not yet clear in rodents (Sawtell et al., 2003; Hensch, 2004; Tagawa et al., 2005; Hofer et al., 2006). We confirmed the presence of long-lasting plasticity in the visual cortices of adult mice. Preliminary results from the present study have been published in an abstract form (Tohmi et al., 2005).

\section{Materials and Methods}

The ethics committee of Niigata University approved the experimental protocols used in the present study. One hundred sixty-six male mice of the C57BL/6 strain (Japan Charles River, Tokyo, Japan) were used for all experiments.

Surgical procedures. Mice were deeply anesthetized using urethane (1.6 $\mathrm{g} / \mathrm{kg}$, i.p.). Throughout the recordings, inhalation of $\mathrm{O}_{2}$ gas was maintained, and rectal temperature was kept at $38^{\circ} \mathrm{C}$ using a silicon rubber heater $(5 \times 10 \mathrm{~cm})$ and a temperature controller (TR-P; AS ONE, Osaka, Japan). The head of each mouse was fixed using a stereotaxic frame (SG-4; Narishige, Tokyo, Japan). Surgical procedures were conducted under sterile conditions, and the skin was disinfected with a solution containing povidone iodine (Meiji Seika, Tokyo, Japan). After subcutaneous injection of bupivacaine (Astra Zeneca, Osaka, Japan), a local anesthetic, disinfected skin was incised, and the skull covering the left and right visual cortices was exposed. The surface of the intact skull was covered with a mixture of liquid paraffin and Vaseline to prevent drying and to keep the skull transparent. Surgical procedures were terminated within $30 \mathrm{~min}$. An additional dose of urethane $(0.2 \mathrm{~g} / \mathrm{kg}$, s.c.) was administered when necessary. Some recording experiments were also performed under anesthesia using pentobarbital ( $60 \mathrm{mg} / \mathrm{kg}$, i.p.). At the end of the recording experiments, mice were killed with an overdose of pentobarbital (intraperitoneally), unless specified otherwise.

Fluorescence imaging. Fluorescence measurements were started $\sim 1 \mathrm{~h}$ after anesthesia. Cortical images $(128 \times 168$ pixels after binning $)$ of endogenous green fluorescence $(\lambda=500-550 \mathrm{~nm})$ in blue light $(\lambda=$ $470-490 \mathrm{~nm}$ ) were recorded in the area containing the left and right visual cortices at nine frames per second by a cooled CCD camera system (AQUACOSMOS/Ratio with ORCA-ER camera; Hamamatsu Photonics, Hamamatsu, Japan) attached to a binocular epifluorescence microscope with a $75 \mathrm{~W}$ xenon light source (MZ FL III; Leica, Nussloch, Germany). Fluorescence images were taken in a recording session, during which mice were given visual stimuli in trials repeated at $20 \mathrm{~s}$ intervals. Images elicited by a particular stimulus were averaged over 24 trials in the experiments shown in Figures $1 A-C, 5 A$, and 7-9. However, the number of trials was increased to 80 in the experiments shown in Figures 2, 4, and 6 and to 120 in the experiments shown in Figure 3A. Spatial averaging in areas of $5 \times 5$ pixels was used to improve image quality. Images were normalized pixel by pixel with respect to a reference image, which was obtained by averaging five images taken immediately before stimulation. The normalized images were shown in a pseudocolor scale representing relative fluorescence changes $\left(\Delta F / F_{0}\right)$. Response amplitude was evaluated as values of $\Delta F / F_{0}$ in a circular window (diameter, $1 \mathrm{~mm}$ ) including the response peak, which was found by visual inspection of serial pseudocolor images $0.6-1.0 \mathrm{~s}$ after stimulus onset. The location of the window was adjusted to maximize response amplitude. In the experiments shown in Figures $1 D$ and 6-9, five images taken between 0.5 and $1.0 \mathrm{~s}$ after stimulus onset were averaged to improve the signal-to-noise ratio. This range was selected to minimize the effects of neurovascular responses on the averaged images or on quantitative analyses. During recording experiments, stimulated eyes were opened until the full extent of the pupil was exposed. Corneas were repeatedly covered with saline to prevent drying throughout the experiments.

Intrinsic signal imaging. Endogenous fluorescence responses were compared with intrinsic signals, which are widely used for functional brain imaging (Grinvald et al., 1986; Frostig et al., 1990; Shtoyerman et al., 2000; Schuett et al., 2002). After completion of flavoprotein fluorescence imaging, the brain was illuminated using a halogen lamp light source via an interference filter passing red light $(\lambda=610 \pm 10 \mathrm{~nm}$; Asahi Bunkou, Tokyo, Japan), and cortical images were directly taken by the same CCD camera used for fluorescence imaging. Because changes in light reflection $\left(\Delta R / R_{0}\right)$ were approximately one-tenth of the fluorescence changes $\left(\Delta F / F_{0}\right)$, images taken in 80 trials were averaged before additional analyses.

Electrophysiology. After imaging experiments, small holes (diameter, 1 $\mathrm{mm}$ ) were made on the skull inside and outside the response areas determined by flavoprotein fluorescence imaging. A tungsten electrode insulated with polyvinyl chloride, except for $30 \mu \mathrm{m}$ from the tip (1-2 M $\Omega$ ), was inserted into the cortex through a hole, and field potentials were recorded at a depth of $0.5 \mathrm{~mm}$ from the cortical surface. Signals were filtered between $0.08 \mathrm{~Hz}$ and $3 \mathrm{kHz}$ and averaged 50 times. A reference electrode was placed in the muscle over the cerebellum.

$M D)$. Mice were anesthetized with ether, and the skin around one eye was disinfected with $70 \%$ alcohol. Eyelids were sutured with a fine surgical Nylon thread (diameter, $0.23 \mathrm{~mm}$; Mani, Tochigi, Japan). During $\mathrm{MD}$, mice were checked daily to make sure that eyes remained closed and uninfected. An ophthalmic solution containing levofloxacin $(5 \mathrm{mg} / \mathrm{ml}$; Santen Pharmaceutical, Osaka, Japan) was applied to the sutured eye every day. When cortical activities were measured before and after MD in the same mice, eyelids were sutured immediately after the first imaging experiment; these were opened again immediately before post-MD imaging experiments. When the imaging experiments were performed a few weeks after MD, the deprived eye was opened under ether anesthesia, and the ophthalmic solution containing levofloxacin was applied to the opened eye.

Visual stimulation. We used two types of visual stimuli. First, a red light-emitting diode (LED) ( $\lambda=613 \mathrm{~nm}$; diameter, $3 \mathrm{~mm}$; TLSH160(F); Toshiba, Tokyo, Japan), which was placed $30 \mathrm{~cm}$ away from the mouse in the horizontal plane, was used in the experiments shown in Figs. 1, 2, 5, 7, 8 , and $9 A$. The direction of the stimulus within the horizontal plane was adjusted. The LED was turned on for $4 \mathrm{~s}$ in each trial. One of the two eyes was covered to enable simulation of the uncovered eye only, except for the experiments shown in Figures $1 D$ and 2, in which both eyes were stimulated.

As a second type of stimulus, various patterned stimuli were produced by a visual stimulus generator (ViSaGe; Cambridge Research System, Cheshire, UK) and shown on a liquid crystal display (8 inch; Chuo Musen, Tokyo, Japan). To avoid perturbation of fluorescence measurements using blue and green lights, the surface of the monitor was covered with a filter passing light with $\lambda>600 \mathrm{~nm}$ (Sharp cut filter; Kenko, Tokyo Japan). When the contrasts of the grating patterns were changed (see Fig. 3 ), the monitor was placed at an angle of $45^{\circ}, 30 \mathrm{~cm}$ away from the mice. Static vertical grating of 0.18 cycle/degree with a square-wave contrast was inverted every $1 \mathrm{~s}$ in the range of $22 \times 18^{\circ}$ (width $\times$ height) for $2 \mathrm{~s}$. When the spatial resolutions of grating patterns were changed (see Fig. 4), the monitor was placed in front of, and $45 \mathrm{~cm}$ away from, the mice. Static vertical grating patterns of a sine-wave contrast between 0.3 and 0.6 cycle/degree were inverted every $1 \mathrm{~s}$ for $2 \mathrm{~s}$.

For the generation of retinotopic maps (see Fig. 6) and the measurement of experience-dependent plasticity in adult mice (Fig. 9 B, C), moving square-wave grating patterns (spatial frequency, 0.18 cycle/degree; speed, 5 cycles/s), randomly changing direction every $0.4 \mathrm{~s}$, were presented to mice for $2 \mathrm{~s}$. Retinotopic maps were produced by moving the monitor with moving square-wave grating patterns in the range of $10 \times$ $18^{\circ}$ (width $\times$ height). When experience-dependent plasticity remaining in adult mice was measured, moving square-wave grating patterns within a range of $22 \times 18^{\circ}$ (width $\times$ height) were presented in front of mice to stimulate responses in the binocular zone of the visual cortex, and at $90^{\circ}$ to stimulate responses in the monocular zone of the visual cortex.

Imaging experiments before and after $M D$ in the same mice. For comparison of cortical activities before and after MD in the same mouse, the surface of the skull of a mouse anesthetized with urethane was cleaned with sterile saline and covered with clear acrylic dental resin (Super bond; Sun Medical, Shiga, Japan). After solidification of the resin, the surface 

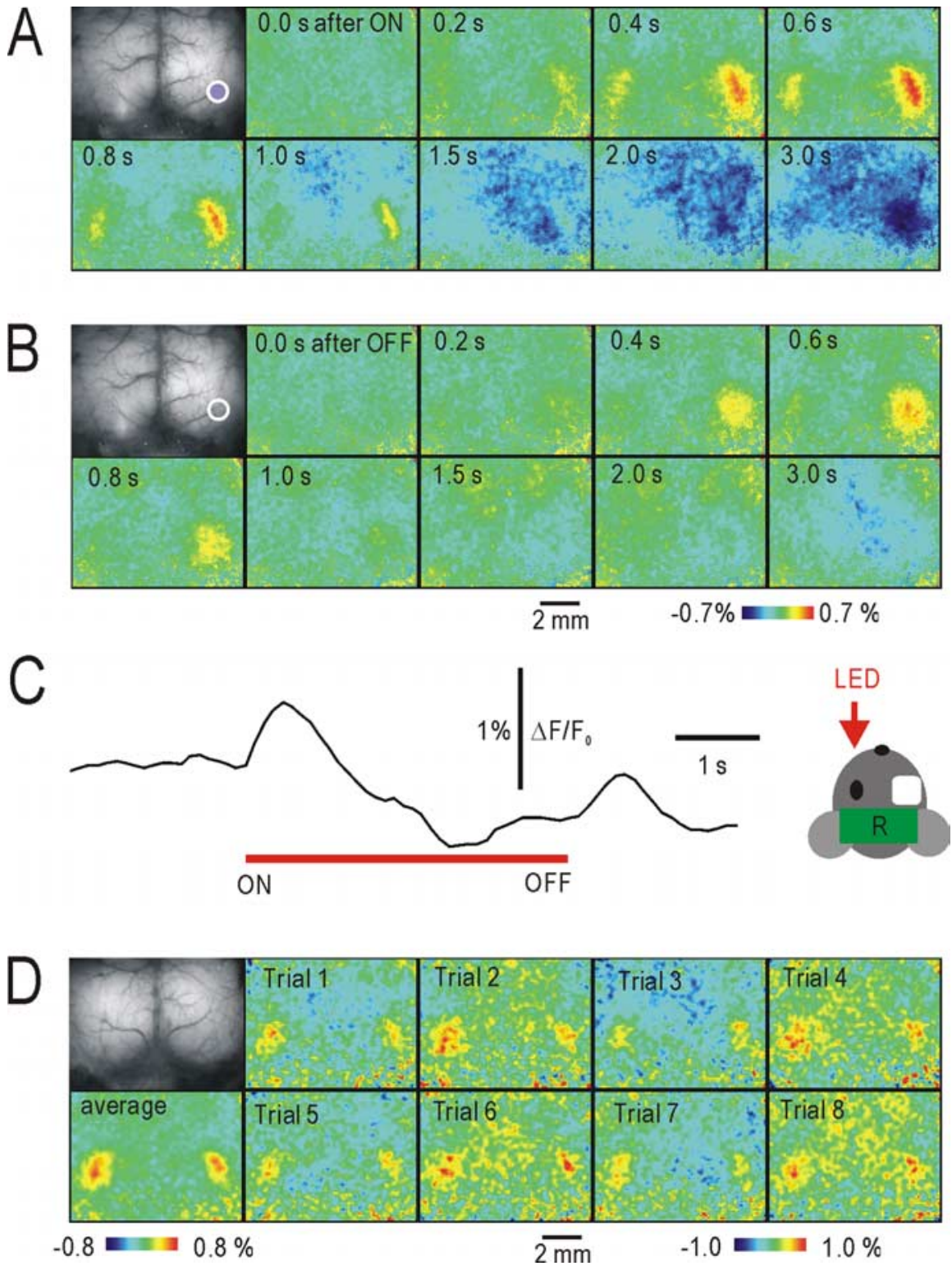

Figure 1. Cortical responses induced by LED light stimuli. $\boldsymbol{A}$, Original fluorescence image and serial pseudocolor images in $\Delta F / F_{0}$ of the left and right visual cortices. In this mouse, the right eye was covered, and an LED light placed in front of the mouse was turned on for $4 \mathrm{~s}$. Time after stimulus onset is shown in each pseudocolor image. Note that on-responses in the contralateral right visual cortex are shown together with faint responses in the ipsilateral left cortex. A reduction in intensity of fluorescence signals, partly attributable to activity-dependent hemodynamic responses, was observed even during the presence of the light stimulus. $\boldsymbol{B}$, Off-responses. A standard image was obtained by averaging five images recorded immediately before offset of the LED light stimulus; serial pseudocolor images were obtained using this standard image. Time after stimulus offset is shown in each pseudocolor image. $\boldsymbol{C}$, Time courses of $\Delta F / F_{0}$ in a circular window in $\boldsymbol{A}$ and $\boldsymbol{B}$ placed on the response center in the contralateral right visual cortex. The red horizontal bar shows the timing of LED light stimulation. Schematic drawings of LED stimulation and recording $(R)$ are also shown. Data shown in $\boldsymbol{A}-\boldsymbol{C}$ were obtained from the same mouse by averaging 24 trials. $\boldsymbol{D}, 0$-responses elicited in a different mouse by the LED stimulus. Each pseudocolor image was obtained from data recorded in eight serial trials repeated at $20 \mathrm{~s}$ intervals; on-responses recorded $0.5-1.0 \mathrm{~s}$ after stimulus onset are shown.

Statistical analysis. The statistical significance of the results was tested using StatView software (SAS Institute, Cary, NC). Differences between unpaired data were evaluated by the MannWhitney $U$ test, and those between paired data were evaluated by the Wilcoxon signed rank test.

\section{Results}

On- and off-responses observed as fluorescence changes in the visual cortex

The skulls of mice are transparent and do not emit endogenous green fluorescence in blue light. Therefore, fluorescence images of the cerebral cortex were taken through the intact skull. When the left eye was stimulated with an LED light placed in front of the mouse, fluorescence responses appeared $0.2 \mathrm{~s}$ after stimulus onset in the contralateral and ipsilateral visual cortices (Fig. 1A), corresponding to the binocular zone in the visual cortex (Dräger, 1975; Schuett et al., 2002). The response peak was observed $0.6-1.0 \mathrm{~s}(0.80 \pm 0.03 \mathrm{~s}$; mean \pm SEM; $n=12$ ) after stimulus onset, and amplitude of the response peak in $\Delta F / F_{0}$ was $0.65 \pm 0.05 \%$. After the response peak, the level of fluorescence gradually decreased below the baseline level observed before stimulus presentation (Fig. 1C). Some of these negative responses were observed around arteries on the cortical surface, suggesting that they were produced by activity-dependent hemodynamic responses (Shibuki et al., 2003). Fluorescence increases were observed after removal of the stimulus in the same places where on-responses appeared (Fig. 1 B.C), suggesting the presence of offresponses in the visual cortex (Hubel and Wiesel, 1962). The response peak was observed $0.78 \pm 0.05 \mathrm{~s}(n=12)$ after stimulus offset, and the amplitude of the response peak in $\Delta F / F_{0}$ was $0.20 \pm 0.03 \%$. Averaging of trials was performed to quantitatively investigate neural activities. However, fluorescence responses elicited by visual stimuli were sufficiently large to be recognized in each trial (Fig. 1D).

\section{Relationship between fluorescence responses and field potentials elicited by visual stimuli}

To investigate the relationship between fluorescence responses and underlying neural activities, we recorded field poten-

was coated with liquid paraffin to reduce random reflection of light. After the first imaging experiments, the eyelid of one eye was sutured. Mice were allowed to recover from anesthesia in a warmed cage. Fradiomycin (Mochida Pharmaceutical, Tokyo, Japan) and ampicillin (Meiji Seika, Tokyo, Japan) were used to avoid infection. When cortical activities were investigated $5 \mathrm{~d}$ after the first imaging experiments, mice were anesthetized with urethane. The surface of the acrylic dental resin covering the skull was washed with sterile saline and covered with liquid paraffin once again. tials in cortical areas showing fluorescence responses (Fig. 2). When both eyes were stimulated by an LED placed in front of the mouse, fluorescence responses appeared in the binocular zone of the visual cortex (Fig. $2 A, B$ ). Negative field potentials were also recorded in the response area identified by the imaging experiment (Fig. 2C). However, no clear potentials were recorded outside the center of the response area (Fig. 2D-F). Fluorescence 

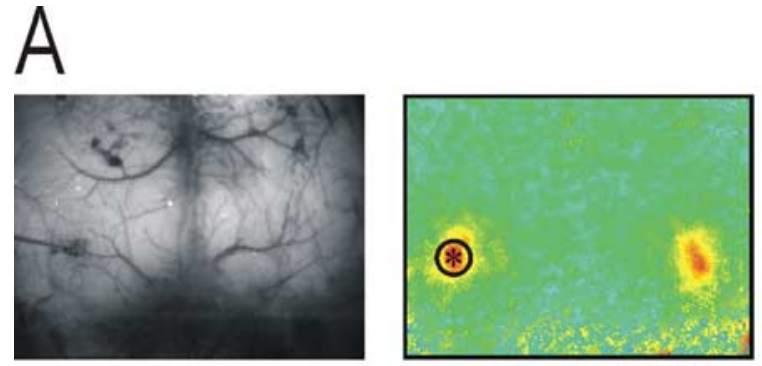

$-2 \mathrm{~mm} \quad-1.0 \%=1.0 \%$
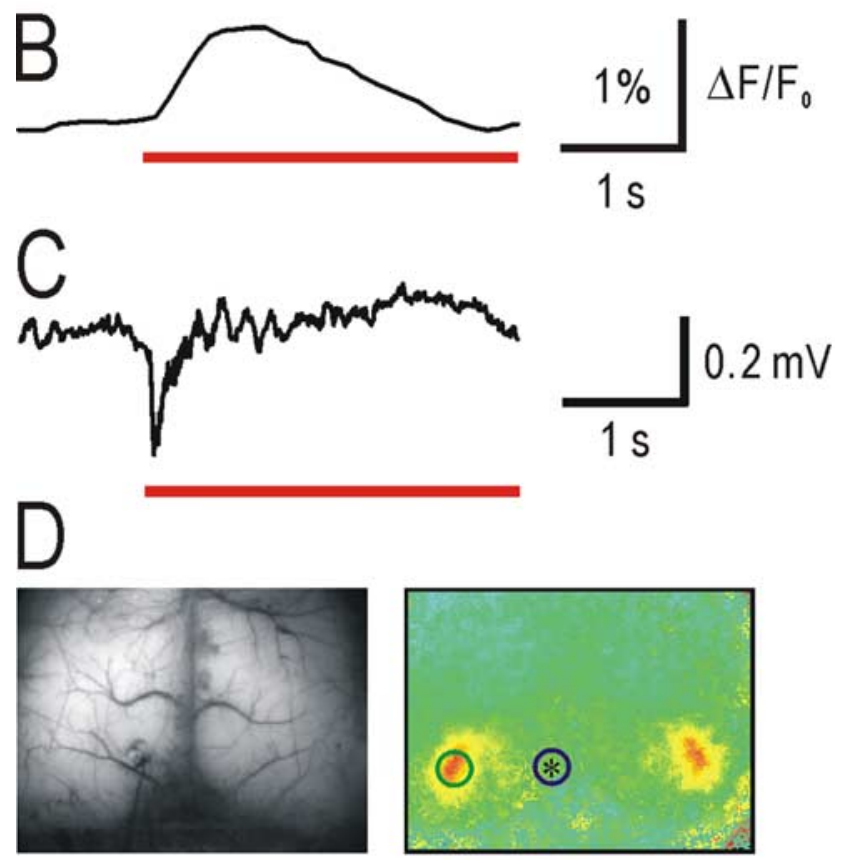

$-2 \mathrm{~mm}$

$-1.0$

$1.0 \%$
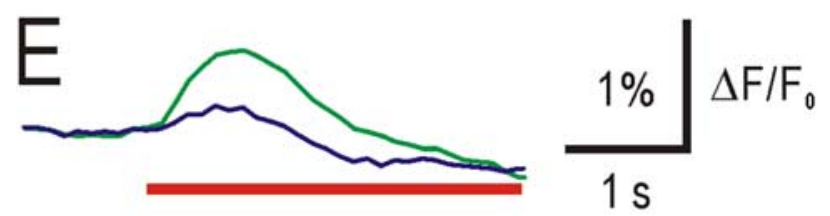

F
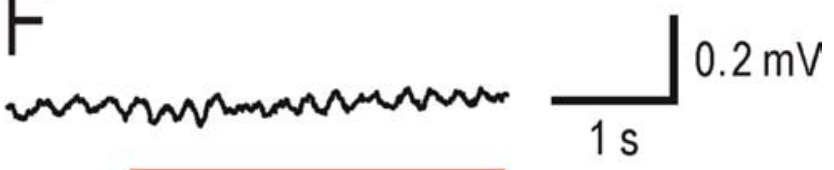

Figure 2. Comparison between flavoprotein fluorescence imaging and field potential recordings. $A$, Original fluorescence image and pseudocolor images in $\Delta F / F_{0}$ observed after stimulus onset. Responses were elicited by LED light stimuli. $\boldsymbol{B}$, Time course of fluorescence changes within a circular window placed at the response center in $\boldsymbol{A}$. $C$, Field potentials recorded at the response center (asterisk in $\boldsymbol{A}$ ). Data in $\boldsymbol{A}-\boldsymbol{C}$ were obtained from the same mouse. $\boldsymbol{D}$, Original fluorescence image and pseudocolor images after stimulus onset. LED light stimuli were provided to both eyes. $\boldsymbol{E}$, Time course of fluorescence changes within the green and blue circular windows in $\boldsymbol{D}$. Colors of traces correspond to those of the windows. $\boldsymbol{F}$, Field potentials recorded outside the response center (asterisk in $\boldsymbol{D}$ ). Data in $\boldsymbol{D}-\boldsymbol{F}$ were obtained from another mouse.

responses showed temporal changes that were slower in time course than field potential responses. Animals anesthetized with urethane sometimes exhibited synchronized slow-wave potentials (Steriade et al., 1993). Remnants of such slow-wave poten-

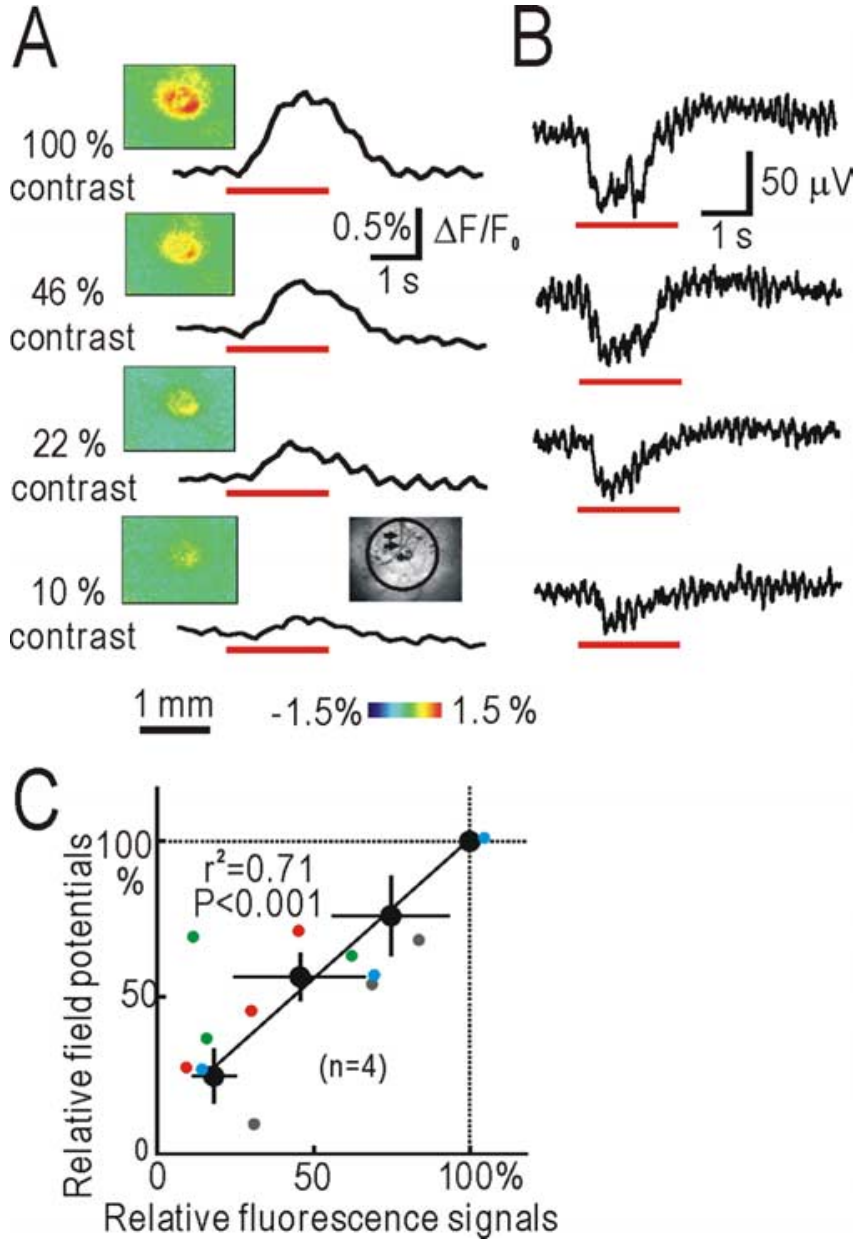

Figure 3. Relationship between fluorescence response and field potentials elicited by grating patterns of various contrasts. $A$, Time course of fluorescence changes elicited by grating patterns in a circular window including the response center in the right cortex contralateral to the stimulated left eye. Fluorescence responses observed after stimulus onset are also shown. Contrasts of grating patterns were varied between 100 and $10 \%$ as shown on the left of each trace. To obtain these data, $80-130$ trials were averaged. $\boldsymbol{B}$, The time course of field potentials recorded through the electrode shown in the original fluorescence image in $\boldsymbol{A}$ (arrows). Fluorescence changes were slightly slower, but the signal-to-noise ratio was comparable or better than that of field potential recordings. $C$, Amplitudes of field potentials plotted against those of fluorescence responses elicited by grating patterns of $100,46.4,21.5$, and $10 \%$ in contrast. Responses were normalized against maximal responses elicited by $100 \%$ contrast patterns in each mouse. The mean and SEM obtained from four mice are shown. A statistically significant correlation $\left(R^{2}=0.71 ; p<0.001\right)$ was observed between the two parameters of the individual data points, which are shown by small dots of different colors.

tials showed a stronger intensity in the averaged field potential traces than in the averaged fluorescence signals, so that the signalto-noise ratio of fluorescence responses was comparable or better than that of field potential recordings. There was also a small increase in fluorescence outside the primary visual cortex, in a region where field potentials showed no modulation with visual stimulation (Fig. 2E,F). This small fluorescence response could reflect nonsynchronized polysynaptic activities, which might not be detected by field potential recordings.

We compared the magnitudes of fluorescence responses and field potential recordings. Visual responses were elicited by grating patterns, in which the contrast of stimuli was varied between 100 and $10 \%$ (Fig. 3). As the contrast of stimuli decreased, the magnitudes of fluorescence responses and field potential responses simultaneously decreased (Fig. $3 A, B$ ). The amplitudes of 


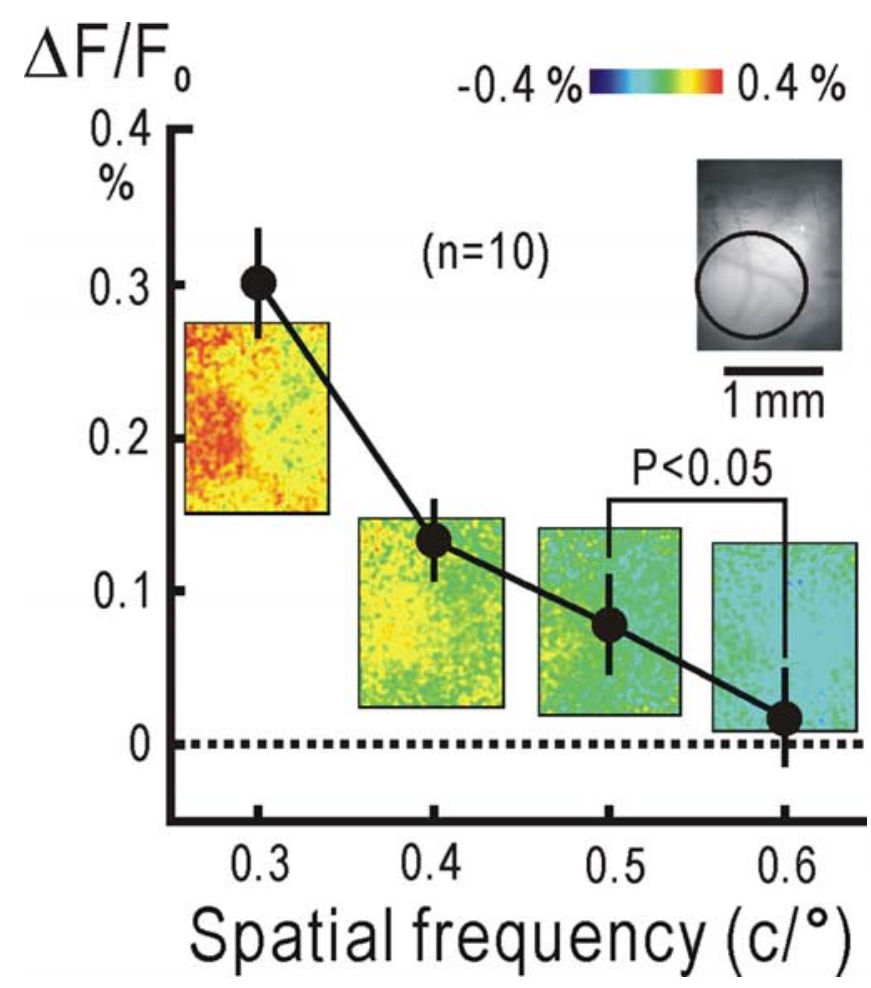

Figure 4. Visual acuity measured by fluorescence responses. The relationship between amplitudes of fluorescence responses measured in a circular window placed on response center and spatial frequencies of the grating patterns used for eliciting responses is shown. The mean and SEM obtained from 10 mice are shown. Examples of pseudocolor images in $\Delta F / F_{0}$ obtained from a mouse are also shown.

fluorescence responses and simultaneously recorded field potentials changed with a significant positive correlation coefficient of 0.71 between the two parameters of the individual data points $(p<0.001)$ (Fig. 3C), indicating that fluorescence responses could be used for quantitative measurement of neural activities.

\section{Estimation of visual acuity using fluorescence responses}

Visual acuity in animals can be determined by behavioral experiments (Prusky et al., 2000; Prusky and Douglas, 2003) or field potential recordings of responses elicited by visual stimuli (Porciatti et al., 1999). We tested whether measurement of fluorescence responses in the visual cortex could be used to estimate visual acuity. Spatial frequency of grating patterns was changed between 0.3 and 0.6 cycle/degree (Fig. 4). Fluorescence responses were clearly observed at a spatial frequency up to 0.5 cycle/degree, whereas almost no response was observed at a spatial frequency of 0.6 cycle/degree. The response amplitude at 0.5 cycle/degree was significantly larger than that at 0.6 cycle/degree $(p<0.05)$, so that the visible frequency limit determined by this method was 0.5 cycle/degree. This value is comparable to the reported visual acuity of $0.5-0.6$ cycle/degree, as determined by behavioral experiments and field potential recordings.

\section{Comparison between flavoprotein fluorescence imaging and intrinsic signal imaging}

Intrinsic signals are produced by differences in red light absorption between oxyhemoglobin and deoxyhemoglobin in capillary blood vessels (Grinvald et al., 1986; Frostig et al., 1990), and intrinsic signal imaging is widely used for functional imaging in the visual cortex (Bonhoeffer and Grinvald, 1991; Schuett et al., 2002; Cang et al., 2005a; Zhan et al., 2005). We compared results obtained using flavoprotein fluorescence imaging (Fig. 5A) and intrinsic signal imaging (Fig. $5 B$ ) in the same mice. Visual stimuli produced fluorescence responses in a similar area of the visual cortex, in which intrinsic signal responses were observed. However, fluorescence images obtained by averaging 24 trials were better in quality than intrinsic signal images obtained by averaging 80 trials, because the amplitude of fluorescence signals $\left(\Delta F / F_{0}\right)$ was $\sim 10$ times larger than that of intrinsic signals $\left(\Delta R / R_{0}\right)$ (Fig. $5 C$ ). Furthermore, the time course of fluorescence signal changes was faster than that of intrinsic signal changes recorded in the same mouse (Fig. $5 C$ ).

\section{Retinotopic maps produced by flavoprotein fluorescence imaging}

Retinotopic maps in the visual cortex have been investigated using electrophysiological recordings (Dräger, 1975; Wagor et al., 1980) and intrinsic signal imaging (Schuett et al., 2002). We produced retinotopic maps using flavoprotein fluorescence imaging. Visual stimuli of moving grating patterns, which were placed between $0^{\circ}$ (front) and $90^{\circ}$ (left) in the horizontal plane, were provided to the left eye. The binocular zone was bilaterally activated by stimuli placed in front of the mouse; when stimuli were placed in other sites, the monocular zone was only activated in the contralateral right cortex (Fig. 6A). In the contralateral right visual cortex, the location of the responsive area was gradually shifted toward the medial side as visual stimuli were laterally shifted in $10^{\circ}$ steps (Fig. $6 \mathrm{~B}$ ). In the lateral area of the primary visual cortex, neural activities were observed in area LM (Schuett et al., 2002). In this area, the retinotopic map was mirrorsymmetrical to those of the primary visual cortex (Fig. 6C), as reported in previous studies (Dräger, 1975; Wagor et al., 1980; Schuett et al., 2002). In rodents, no orientation column has been found in the visual cortex (Antonini et al., 1999; Ohki et al., 2005). We found no heterogeneity in the responsive area stimulated with moving grating patterns of various orientations (data not shown).

\section{Experience-dependent plasticity visualized with flavoprotein} fluorescence imaging

MD in young mice reduces responsivity in the binocular region of the visual cortex to stimuli provided to the deprived eye (Dräger, 1978; Gordon and Stryker, 1996). We attempted to visualize plasticity induced by MD using transcranial flavoprotein fluorescence imaging. Once MD started on postnatal day 28 (P28) for $4 \mathrm{~d}$, bilateral cortical responses to LED light stimuli placed in front of the mouse were recorded. When stimuli were provided to the nondeprived eye, clear responses were observed in both the contralateral and ipsilateral cortices (Fig. 7Aa). When stimuli were provided to the deprived eye, responses in both the contralateral and ipsilateral cortices were clearly depressed (Fig. $7 A c)$. However, no apparent change was observed in the monocular zone (Fig. 7Ab,Ad). In the binocular zone of the contralateral visual cortex, deprived eye responses were significantly reduced compared with nondeprived eye responses $(p<0.003)$ (Fig. $7 B)$. Significant differences were also observed in the binocular zone of the ipsilateral visual cortex $(p<0.02)$ (Fig. $7 B)$. However, no significant difference was observed in the monocular zone. To identify the period of sensitivity to $\mathrm{MD}$, it was initiated at various ages between 3 and 6 weeks old for $4 \mathrm{~d}$. Consistent with previously reported electrophysiological studies (Gordon and Stryker, 1996), the difference in $\Delta F / F_{0}$ between nondeprived eye responses and deprived eye responses was maximal when MD was begun at the age of 4 weeks, in both the contralateral and ipsilat- 
eral visual cortices (Fig. $7 \mathrm{Ca}, \mathrm{Cc}$ ). No effect was found for MD begun at the age of 6 weeks. The duration of MD commenced at 4 weeks of age was varied between 1 and $7 \mathrm{~d}$ (Fig. $7 C b, C d$ ). Although MD for $1-2 \mathrm{~d}$ was not effective, MD for $4 \mathrm{~d}$ was just as effective as that for $7 \mathrm{~d}$.

In previous studies, the cortical responses to stimulation of the ipsilateral and contralateral eyes were compared with evaluate the effects of MD (Dräger, 1978; Gordon and Stryker, 1996). Therefore, we calculated the ratio of $\Delta F / F_{0}$ elicited by stimulation of the ipsilateral eye and that elicited by stimulation of the contralateral eye. The data represented in Figure $7 B$ were used for this calculation, except for that from two mice in which $\Delta F / F_{0}$ of the contralateral deprived eye response was too small $\left(\Delta F / F_{0}<0.2\right)$. The ratio of ipsilateral deprived eye response/contralateral nondeprived eye response $(0.55 \pm 0.06$; $n=13$ ) was significantly smaller than that of ipsilateral nondeprived eye response/ contralateral deprived eye response $(0.97 \pm 0.06 ; p<0.002)$. These findings are compatible with previous reports and suggest that transcranial flavoprotein fluorescence imaging is applicable for investigating experience-dependent plasticity in the visual cortex.

Transcranial imaging preserves the skull. When the surface of the skull is covered with transparent dental resin, the skull is protected against infection and maintains its transparency for several days after surgery (Takao et al., 2006). Using this technique, we compared fluorescence responses in the visual cortex before and after MD in the same mouse. As shown in Figure $8 \mathrm{~A}$, fluorescence responses in the visual cortex were clearly observed before and after MD at $5 \mathrm{~d}$, although some endogenous fluorescence appeared along cranial sutures $5 \mathrm{~d}$ after surgery (Fig. $8 \mathrm{~A}$ ). Nondeprived eye responses increased after $\mathrm{MD}$, whereas deprived eye responses decreased after MD (Fig. $8 A$ ). In the contralateral cortex, increases in nondeprived eye responses $(p<0.05)$ and decreases in deprived eye responses $(p<0.0003)$ were statistically significant (Fig. $8 C)$. In the ipsilateral cortex, decreases in deprived eye responses were significant $(p<0.05)$. As a result, amplitudes of responses elicited via each eye were significantly different after MD for both the contralateral $(p<0.0002)$ and ipsilateral $(p<0.04)$ responses (Fig. $8 C$ ). No significant change was observed in the responses recorded in the monocular zone (Fig. $8 B, C$ ).

\section{Long-lasting effects of MD}

In animals other than mice, experience-dependent plasticity induced by MD in the visual cortex during a critical period is maintained after maturation of the animals (Giffin and Mitchell, 1978; Murphy and Mitchell, 1987). In behavioral studies using mice, it has been demonstrated that reduction in visual acuity produced by MD performed during a critical period is maintained in adult mice (Prusky and Douglas, 2003). However, it is not clear whether experience-dependent plasticity produced by MD during a critical period is preserved in adult mice. To clarify this, we investigated the effects of MD for $7 \mathrm{~d}$ performed between $\mathrm{P} 26$ and P33, in 6-week-old mice. When LED light stimuli were used, no clear effect of MD was found in either the contralateral or ipsilateral visual cortices in 6-week-old mice (Fig. 9Aa, $A b$ ). However, when the effects of MD were investigated with moving grating patterns, differences between deprived eye and nondeprived eye responses were observed in the binocular zone of the contralateral and ipsilateral cortices (Fig. 9Ba,Bc), whereas no clear change was observed in the monocular zone (Fig. $9 B b, B d$ ). The effects of MD were significant in both the contralateral $(p<0.003)$ and ipsilateral $(p<0.006)$ cortices in 6-week-old mice, but no significant effect was observed in the monocular zone (Fig. 9C). These findings indicate that the effects of MD performed during a critical period were preserved in visual responses elicited by complex patterned stimuli but not by simple LED light stimuli.

\section{Discussion}

Transcranial flavoprotein fluorescence imaging in the mouse visual cortex

In the present study, MD-induced plasticity in the mouse visual cortex was investigated using flavoprotein fluorescence imaging. 


\section{$\mathrm{Aa}$}

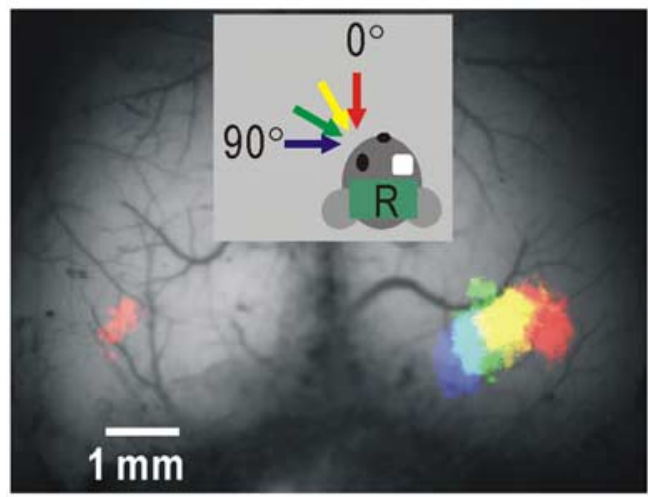

b
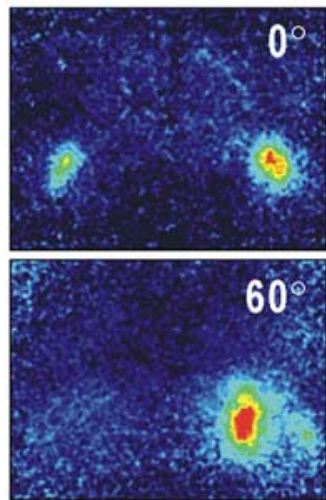

$\overline{2 \mathrm{~mm}}$

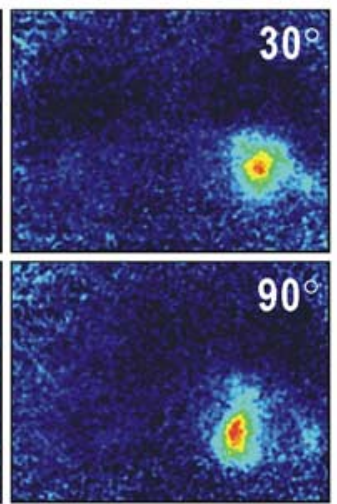

$0 \%=0.8 \%$
B

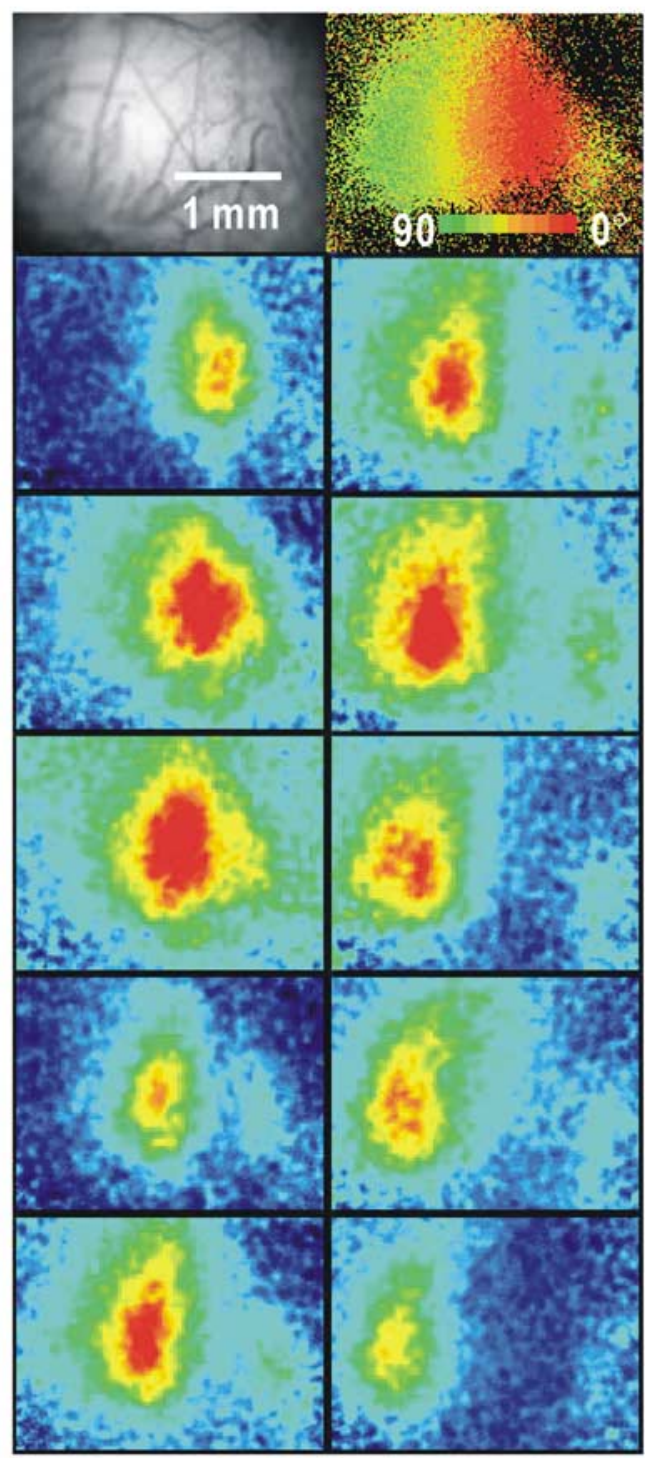

$0 \%=1.2 \%$

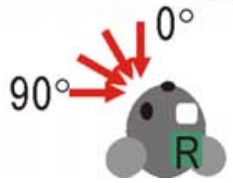

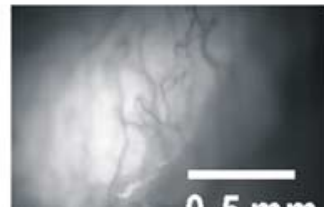

$0.5 \mathrm{~mm}$
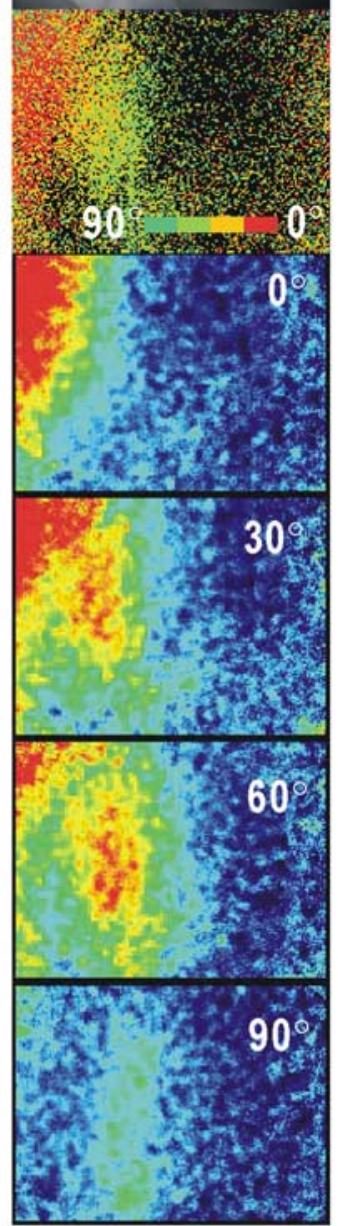

$0 \%=0.4 \%$

Figure 6. Retinotopic maps. Aa, Retinotopic map superimposed on an original fluorescence image. It was produced from fluorescence responses elicited via the left eye using LED stimuli placed at different locations, which are shown with arrows in the inset. The colors of the arrows correspond to those in the map. $\boldsymbol{A} \boldsymbol{b}, 0$ riginal fluorescence responses used to construct the map shown in $\boldsymbol{A} \boldsymbol{a}$. Cortical areas in which $\Delta F / F_{0}$ was larger than half the amplitude of endogenous fluorescence responses in each image in $\boldsymbol{A} \boldsymbol{b}$ were superimposed on the original fluorescence image. $\boldsymbol{B}$, Fine retinotopic map produced from fluorescence responses in the right visual cortex contralateral to the stimulated eye. The topmost panels are the original fluorescence image and the retinotopic map reconstructed using Scion Image (developed by W. Rasband and available from the Internet). In this map, the locations of stimuli producing maximal responses were determined for each pixel and are shown as a pseudocolor image. When amplitudes in $\Delta F / F_{0}$ did not reach $40 \%$ of peak amplitudes, the pixel is shown in black. 0 ther panels show pseudocolor images of visual responses produced by stimuli at various locations. Averaged results of 80 trials are shown. Activities in area LM can be recognized lateral to responses in the primary visual cortex. $C$, Retinotopic map in area LM. $R$, Recording.

A merit of this technique is that variation in recording data, either between each side of the cortex or between animals, is small enough for investigating plastic changes in neural activities. Short-term potentiation and depression induced by direct cortical stimulation with tetanic stimulation have been demonstrated in the rat somatosensory cortex (Murakami et al., 2004). Longterm cortical depression induced by discrimination learning in the rat somatosensory cortex has also been demonstrated (Shibuki et al., 2006b). In mice, the transparent skull allows transcranial imaging, and this technique is used for visualizing cortical activities in the auditory, somatosensory, and visual cortices (Shibuki et al., 2006a). In this case, surgical damage to the brain is minimal, so that reproducible and stable recording is possible. Using this technique, experience-dependent plasticity 

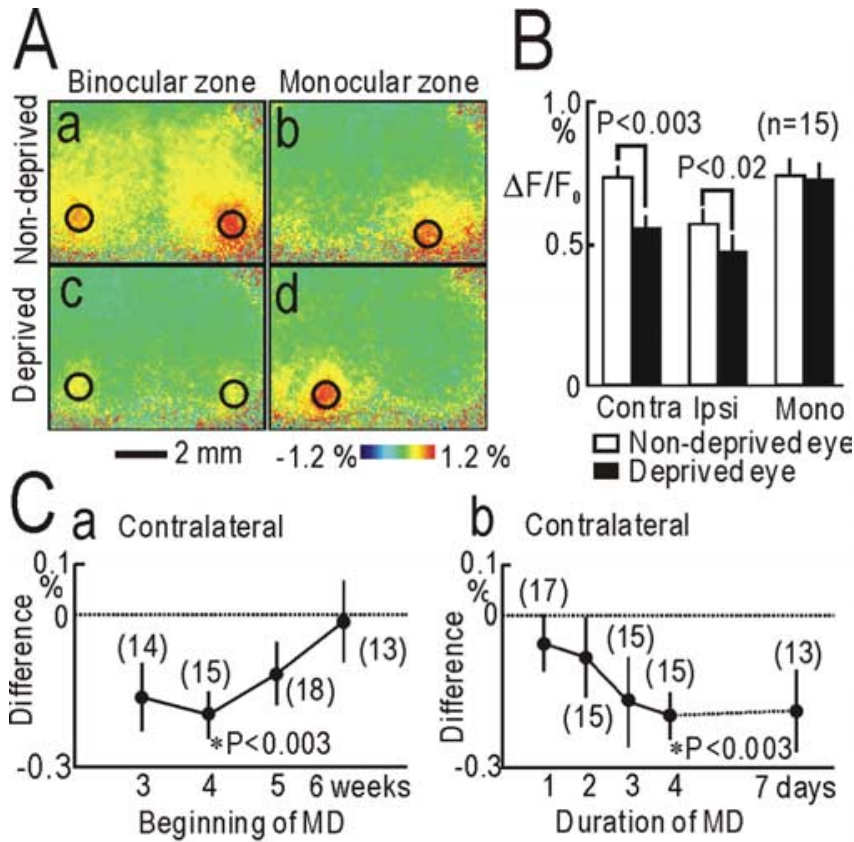

b contralateral

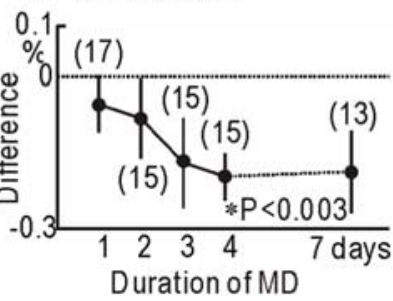

C Ipsilateral
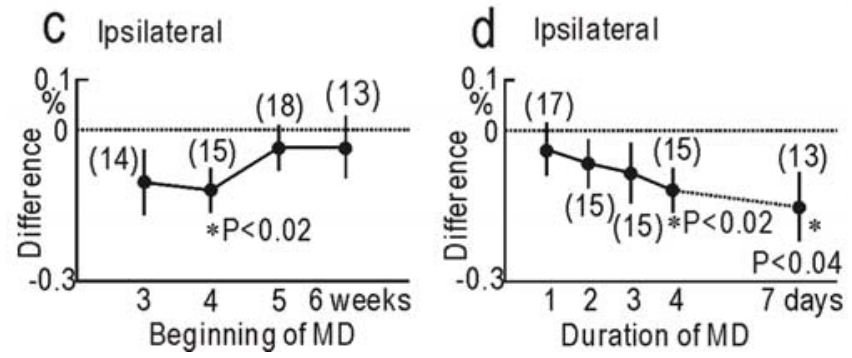

Figure 7. Experience-dependent plasticity induced by MD. A, Fluorescence responses elicited by LED light stimuli in the visual cortex after MD. $\boldsymbol{A a}-\boldsymbol{A d}$, Nondeprived eye responses in the binocular zone $(\boldsymbol{A} \boldsymbol{a})$ and monocular zone $(\boldsymbol{A} \boldsymbol{b})$ and deprived eye responses in the binocular zone (Ac) and monocular zone (Ad). These images were obtained from the same mouse. $\boldsymbol{B}$, Amplitudes of fluorescence responses $\left(\Delta F / F_{0}\right)$ in the contralateral binocular zone (Contra), ipsilateral binocular zone (Ipsi), and contralateral monocular zone (Mono). The mean and SEM from 15 mice are shown. $C a$, Difference in $\Delta F / F_{0}$ between nondeprived eye responses and deprived eye responses in the contralateral binocular zone. MD for $4 \mathrm{~d}$ was started at an age of $3-6$ weeks. Cb, Difference in the contralateral binocular zone. MD for 1-7 d was started at the age of 4 weeks. (c, Difference in the ipsilateral binocular zone. MD for $4 \mathrm{~d}$ was started at $3-6$ weeks of age. $C d$, Difference in the ipsilateral binocular zone. MD for $1-7 d$ was started at 4 weeks of age. Numbers of experiments are shown in parentheses.

has been demonstrated in the mouse auditory (Takahashi et al., 2006) and somatosensory (Kitaura et al., 2005) cortices and in cortical activities during audiogenic seizures in mice (Takao et al., 2006). These previous studies and the present findings clearly indicate the usefulness of transcranial flavoprotein fluorescence imaging for investigating experience-dependent cortical plasticity in mice.

\section{Comparison between fluorescence signals and neural activities in the visual cortex}

In the present study, we compared fluorescence signals recorded in the visual cortex with corresponding field potentials or intrinsic signals, to verify whether the former reflected neural activities in the visual cortex. On- and off-responses in visual cortical neurons (Hubel and Wiesel, 1962) were observed as changes in fluorescence signals (Fig. 1). Field potentials were recorded only in the area where fluorescence responses were observed (Fig. 2). A good correlation was found between fluorescence responses and field potentials elicited by grating patterns of various contrasts

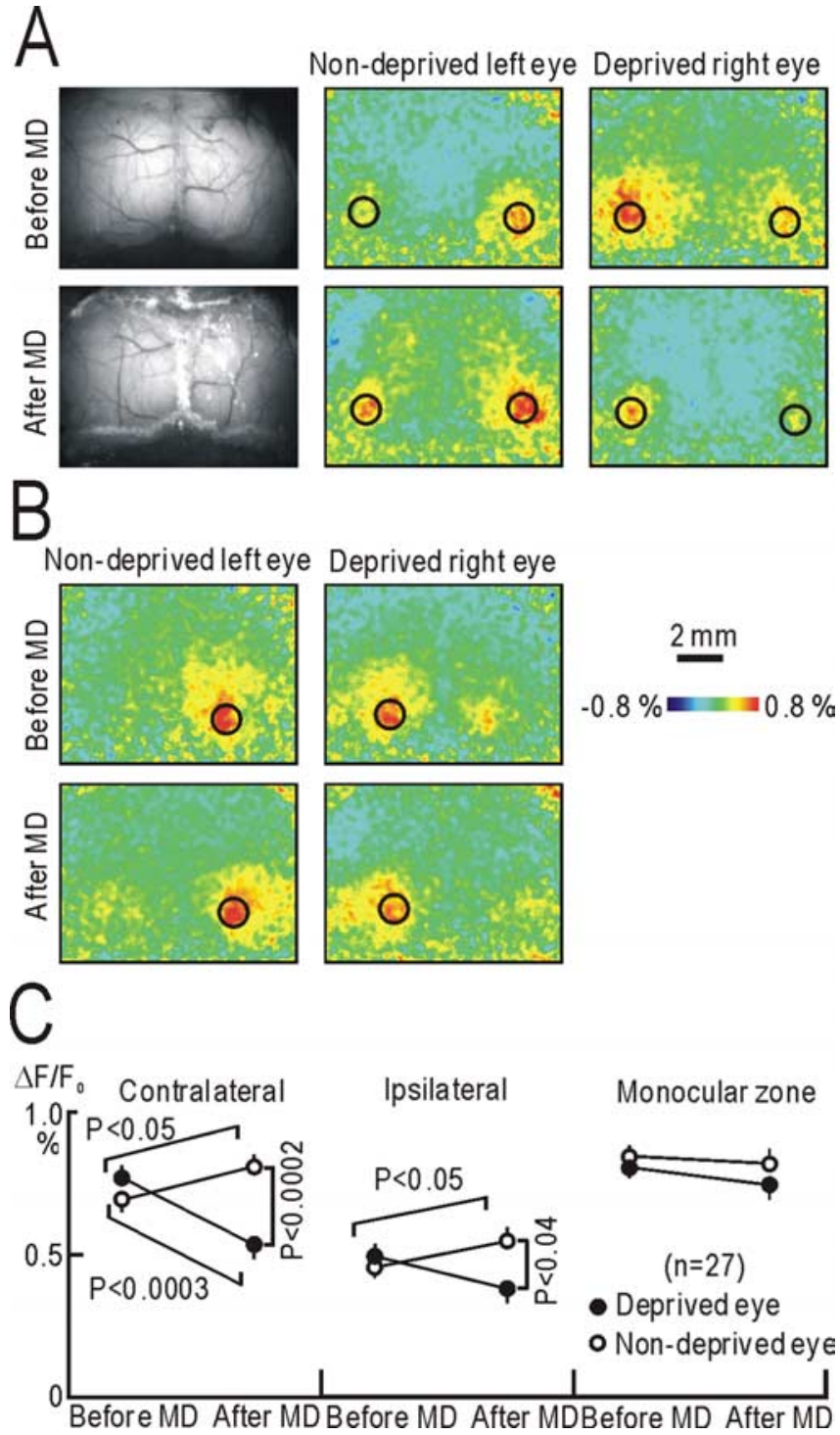

Figure 8. Comparison between fluorescence responses in the binocular zone before and after MD in the same mice. $\boldsymbol{A}$, Responses recorded in the binocular zone. Original fluorescence images before and after MD for $5 \mathrm{~d}$, nondeprived (left) eye responses before and after MD, and deprived (right) eye responses before and after MD are shown. $\boldsymbol{B}$, Responses recorded in the monocular zone. Nondeprived (left) eye responses before and after MD and deprived (right) eye responses before and after $M D$ are shown. Data in $\boldsymbol{A}$ and $\boldsymbol{B}$ were obtained from the same mouse. C, Amplitudes of fluorescence responses in the contralateral binocular zone, ipsilateral binocular zone, and monocular zone before and after MD of $5 \mathrm{~d}$ in the same 27 mice.

(Fig. 3). Visual acuity of mice to grating patterns was investigated, revealing a detection limit of 0.5 cycle/degree (Fig. 4), which was comparable to the value of $0.5-0.6$ cycle/degree determined by behavioral experiments (Prusky et al., 2000; Prusky and Douglas, 2003) and field potential recordings (Porciatti et al., 1999). Fluorescence signals appeared faster than intrinsic signals after stimulus onset (Fig. 5). Fine retinotopic maps could be obtained using fluorescence imaging (Fig. 6). Together, it is strongly suggested that flavoprotein fluorescence imaging is applicable for investigating both the magnitude and spatial distribution of cortical activities in the mouse visual cortex.

Experience-dependent plasticity visualized using flavoprotein fluorescence imaging

The main purpose of the present study was to investigate MDinduced plasticity in the mouse visual cortex. We found that 

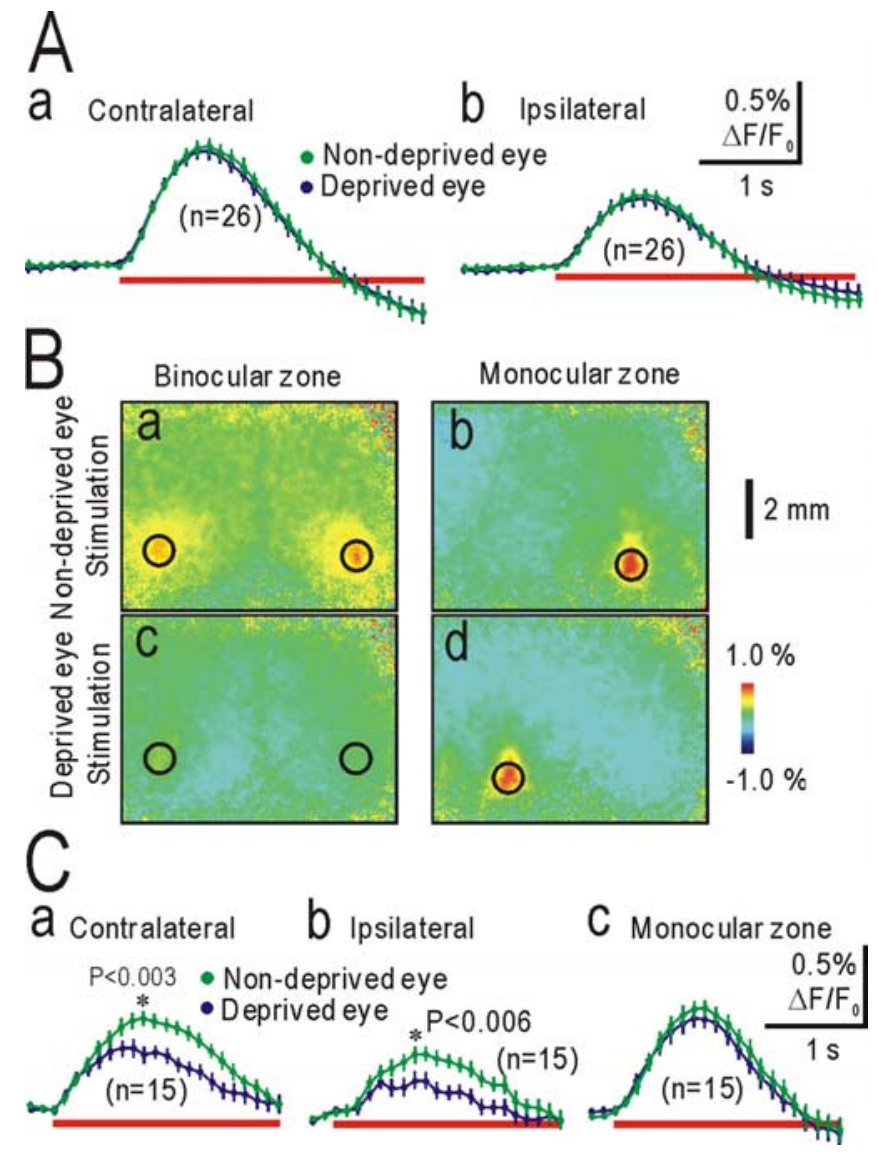

Figure 9. Long-lasting effects of MD performed in young mice. $A$, Time course of fluorescence responses elicited by LED light stimuli in the contralateral $(\boldsymbol{a})$ and ipsilateral $(\boldsymbol{b})$ visual cortices 2 weeks after MD, which was performed between P26 and P33. No apparent difference was observed between nondeprived eye responses and deprived eye responses. $\boldsymbol{B}$, Fluorescence responses elicited by moving grating patterns 2 weeks after MD performed between $\mathrm{P} 26$ and P33. $\boldsymbol{B} \boldsymbol{a}-\boldsymbol{B d}$, Nondeprived eye responses in the binocular zone $(\boldsymbol{B a})$ and the monocular zone $(\boldsymbol{B} \boldsymbol{b})$ and deprived eye responses in the binocular zone $(\boldsymbol{B C})$ and the monocular zone $(\boldsymbol{B} \boldsymbol{d})$ are shown. These images were obtained from the same mouse. Deprived eye responses were reduced after MD compared with nondeprived eye responses in the binocular zone, whereas those in the monocular zone were not. $C$, Time course of fluorescence responses elicited by moving grating patterns in the contralateral $(\boldsymbol{a})$ and ipsilateral $(\boldsymbol{b})$ binocular zones and the contralateral monocular zone (c) of the visual cortex 2 weeks after MD, performed between P26 and P33.

decreases in deprived eye responses compared with nondeprived eye responses occurred in the binocular zone but not in the monocular zone of the visual cortex (Fig. 7). Effects of MD were most clearly observed when started at 4 weeks old and were saturated at the end of $4 \mathrm{~d}$. These characteristics are compatible with previous results obtained using electrophysiological recordings in mice (Dräger, 1978; Gordon and Stryker, 1996). Using intrinsic signal imaging, MD-induced plasticity has been observed in mice anesthetized with pentobarbital but not in mice anesthetized with urethane (Cang et al., 2005a). We demonstrated MD-induced plasticity in mice anesthetized with urethane using flavoprotein fluorescence responses (Fig. 7). We performed experiments in mice anesthetized with urethane, because the long-lasting effect of urethane was suitable for stable recordings of neural activities, although MD-induced plasticity could also be observed in mice anesthetized with pentobarbital (supplemental Fig. 1, available at www.jneurosci.org as supplemental material). A discrepancy between intrinsic signal imaging and flavoprotein fluorescence imaging might be explained by some quantitative aspects of neural activities being obscured in intrinsic signal imaging, which might not reflect neural activities including magnitude (Nemoto et al., 2004; Sheth et al., 2004; Zhan et al., 2005).

A technical merit of flavoprotein fluorescence imaging is that neural activities in a wide area including bilateral visual cortices can be observed simultaneously (Fig. 7), so that fluorescence responses elicited via the deprived eye in the right/left cortex and those elicited via the nondeprived eye in the left/right cortex can be compared directly. Responses in the monocular zone can be easily recorded as a control. Furthermore, when the surface of the skull was covered with clear acrylic resin, responses elicited via the deprived eye and those elicited via the nondeprived eye could be compared before and after MD in the same mice (Fig. 8A,B). From such analyses, we concluded that MD-induced plasticity in young mice has two components: potentiation of nondeprived eye responses and depression of deprived eye responses (Fig. 8C). This conclusion has also been reached regarding contralateral deprived eye responses and ipsilateral nondeprived eye responses, in a previous study of field potential recordings in mice (Frenkel and Bear, 2004). In the present study, bilateral cortical responses were observed in the same mice, and depression of contralateral deprived eye responses, potentiation of contralateral nondeprived eye responses, and potentiation of ipsilateral nondeprived eye responses were found (Fig. $8 C$ ). Ipsilateral deprived eye responses were also depressed, but the changes were not statistically significant.

\section{Long-lasting effects of MD in adult mice}

In the present study, effects of MD performed during a critical period were detected in adult mice (Fig. 9) as reported in other animals (Giffin and Mitchell, 1978; Murphy and Mitchell, 1987). Prior MD during a critical period enhances plasticity in the adult visual cortex visualized with intrinsic signal imaging (Hofer et al., 2006); however, long-lasting effects of MD after reopening of the deprived eye have not been previously observed by electrophysiological recordings in rodents (Sawtell et al., 2003; Hensch, 2004; Tagawa et al., 2005). We confirmed long-lasting plasticity when moving grating patterns were used as visual stimuli (Fig. 9B,C), whereas no long-lasting effect of MD was found in responses elicited by LED light stimuli (Fig. 8). Our findings are compatible with a behavioral study showing that reduction in visual acuity in the deprived eye caused by MD during a critical period is maintained in adult mice (Prusky and Douglas, 2003). The difference between LED light stimuli and moving grating patterns can probably be explained by the fact that LED light stimuli might be appropriate for activating thalamocortical synapses, but might not be suitable for activating intracortical circuits. Response properties of cortical neurons are more complex than those of thalamic relay neurons in the lateral geniculate body (Hubel and Wiesel, 1961, 1962; Dräger, 1975), so that intracortical circuits can be effectively stimulated by moving grating patterns but not by LED light stimuli. Therefore, the long-lasting effects of MD might be observed only in intracortical circuits, but not in thalamocortical synapses. In accordance with this idea, MDinduced plasticity is more clearly observed in supragranular and infragranular neurons than in layer IV (Gordon and Stryker, 1996), suggesting the presence of MD-induced plasticity in intracortical circuits connecting layer IV and other layers.

\section{Future applications of flavoprotein fluorescence imaging in the mouse visual cortex}

The molecular mechanisms of MD-induced plasticity have been investigated intensively using various strains of mice lacking specific genes (Huang et al., 1999; Taha et al., 2002; Sawtell et al., 
2003; Mataga et al., 2004; Cang et al., 2005b; McGee et al., 2005). Obviously, one of the most promising possibilities of flavoprotein fluorescence imaging is its potential application to various strains of genetically manipulated mice, because a large number of mice can be efficiently investigated using this technique. A clear critical period for MD-induced plasticity has been demonstrated in previous studies using single-unit recordings in mice (Gordon and Stryker, 1996), but the presence of a similar critical period has not been demonstrated in studies using other methods (Tagawa et al., 2005; Sawtell et al., 2003). In the present study, a critical period comparable to that obtained using single-unit recordings was demonstrated under urethane anesthesia (Fig. 7C); this result might be helpful for clarifying the mechanisms underlying the difference in the critical periods obtained using different methods. When spontaneous slow-wave oscillation (Steriade et al., 1993) was not observed, flavoprotein fluorescence imaging was sufficient to visualize cortical activities without averaging trials (Fig. 1D). Trial-to-trial variability of cortical responses, which has been investigated using voltage-sensitive dyes (Arieli et al., 1996), can also be investigated using flavoprotein fluorescence imaging. In the present study, we focused on visualizing cortical activities in a wide area including bilateral visual cortices. However, activity-dependent changes in NADH signals have been demonstrated at the single-cell level in slice experiments using two-photon microscopy (Kasischke et al., 2004). Furthermore, in vivo transcranial imaging has been used to visualize fine morphological changes in a single neuron using two-photon microscopy (Grutzendler et al., 2002; Ohki et al., 2005; Zuo et al., 2005). Therefore, there would be no spatial limitation to investigate neural activities at the single-cell level using transcranial flavoprotein fluorescence imaging in mice. A combination of transcranial flavoprotein fluorescence imaging and two-photon microscopy might be applicable to the investigation of the fine cellular mechanisms underlying experience-dependent plasticity in the mouse visual cortex.

\section{References}

Antonini A, Fagiolini M, Stryker MP (1999) Anatomical correlates of functional plasticity in mouse visual cortex. J Neurosci 19:4388-4406.

Arieli A, Sterkin A, Grinvald A, Aertsen A (1996) Dynamics of ongoing activity: explanation of the large variability in evoked cortical responses. Science 273:1868-1871.

Bonhoeffer T, Grinvald A (1991) Iso-orientation domains in cat visual cortex are arranged in pinwheel-like patterns. Nature 353:429-431.

Cang J, Kalatsky VA, Lowel S, Stryker MP (2005a) Optical imaging of the intrinsic signal as a measure of cortical plasticity in the mouse. Vis Neurosci 22:685-691.

Cang J, Kaneko M, Yamada J, Woods G, Stryker MP, Feldheim DA (2005b) Ephrin-as guide the formation of functional maps in the visual cortex. Neuron 48:577-589.

Chance B, Cohen P, Jöbsis FF, Schoener B (1962) Intracellular oxidationreduction states in vivo. Science 137:499-508.

Coutinho V, Mutoh H, Knöpfel T (2004) Functional topology of the mossy fibre-granule cell-Purkinje cell system revealed by imaging of intrinsic fluorescence in mouse cerebellum. Eur J Neurosci 20:740-748.

Dräger UC (1975) Receptive fields of single cells and topography in mouse visual cortex. J Comp Neurol 160:269-290.

Dräger UC (1978) Observations on monocular deprivation in mice. J Neurophysiol 41:28-42.

Frenkel MY, Bear MF (2004) How monocular deprivation shifts ocular dominance in visual cortex of young mice. Neuron 44:917-923.

Frostig RD, Lieke EE, Ts'o DY, Grinvald A (1990) Cortical functional architecture and local coupling between neuronal activity and the microcirculation revealed by in vivo high-resolution optical imaging of intrinsic signals. Proc Natl Acad Sci USA 87:6082-6086.

Giffin F, Mitchell DE (1978) The rate of recovery of vision after early monocular deprivation in kittens. J Physiol (Lond) 274:511-537.
Gordon JA, Stryker MP (1996) Experience-dependent plasticity of binocular responses in the primary visual cortex of the mouse. J Neurosci 16:3274-3286

Grinvald A, Lieke E, Frostig RD, Gilbert CD, Wiesel TN (1986) Functional architecture of cortex revealed by optical imaging of intrinsic signals. Nature 324:361-364.

Grutzendler J, Kasthuri N, Gan WB (2002) Long-term dendritic spine stability in the adult cortex. Nature 420:812-816.

Hensch TK (2004) Critical period regulation. Annu Rev Neurosci 27:549-579.

Hofer SB, Mrsic-Flogel TD, Bonhoeffer T, Hübener M (2006) Prior experience enhances plasticity in adult visual cortex. Nat Neurosci 9:127-132.

Huang ZJ, Kirkwood A, Pizzorusso T, Porciatti V, Morales B, Bear MF, Maffei L, Tonegawa S (1999) BDNF regulates the maturation of inhibition and the critical period of plasticity in mouse visual cortex. Cell 98:739-755.

Hubel DH, Wiesel TN (1961) Integrative action in the cat's lateral geniculate body. J Physiol (Lond) 155:385-398

Hubel DH, Wiesel TN (1962) Receptive fields, binocular interaction and functional architecture in the cat's visual cortex. J Physiol (Lond) 160:106-154.

Hubel DH, Wiesel TN (1970) The period of susceptibility to the physiological effects of unilateral eye closure in kittens. J Physiol (Lond) 206:419-436.

Hubel DH, Wiesel TN, LeVay S (1977) Plasticity of ocular dominance columns in monkey striate cortex. Philos Trans R Soc Lond B Biol Sci 278:377-409.

Kalatsky VA, Stryker MP (2003) New paradigm for optical imaging: temporally encoded maps of intrinsic signal. Neuron 38:529-545.

Kasischke KA, Vishwasrao HD, Fisher PJ, Zipfel WR, Webb WW (2004) Neural activity triggers neuronal oxidative metabolism followed by astrocytic glycolysis. Science 305:99-103.

Kitaura H, Ikeda K, Takahashi K, Tohmi M, Kudoh M, Shibuki K (2005) Somatotopic map reorganization after tail amputation in mouse primary somatosensory cortex visualized using transcranial flavoprotein autofluorescence imaging. Soc Neurosci Abstr 31:174.11.

LeVay S, Wiesel TN, Hubel DH (1980) The development of ocular dominance columns in normal and visually deprived monkeys. J Comp Neurol 191:1-51.

Mataga N, Mizuguchi Y, Hensch TK (2004) Experience-dependent pruning of dendritic spines in visual cortex by tissue plasminogen activator. Neuron 44:1031-1041.

McGee AW, Yang Y, Fischer QS, Daw NW, Strittmatter SM (2005) Experience-driven plasticity of visual cortex limited by myelin and Nogo receptor. Science 309:2222-2226.

Murakami H, Kamatani D, Hishida R, Takao T, Kudoh M, Kawaguchi T, Tanaka R, Shibuki K (2004) Short-term plasticity visualized with flavoprotein autofluorescence in the somatosensory cortex of anesthetized rats. Eur J Neurosci 19:1352-1360.

Murphy KM, Mitchell DE (1987) Reduced visual acuity in both eyes of monocularly deprived kittens following a short or long period of reverse occlusion. J Neurosci 7:1526-1536.

Nemoto M, Sheth S, Guiou M, Pouratian N, Chen JW, Toga AW (2004) Functional signal- and paradigm-dependent linear relationships between synaptic activity and hemodynamic responses in rat somatosensory cortex. J Neurosci 24:3850-3861.

Ohki K, Chung S, Ch'ng YH, Kara P, Reid RC (2005) Functional imaging with cellular resolution reveals precise micro-architecture in visual cortex. Nature 433:597-603.

Porciatti V, Pizzorusso T, Maffei L (1999) The visual physiology of the wild type mouse determined with pattern VEPs. Vision Res 39:3071-3081.

Prusky GT, Douglas RM (2003) Developmental plasticity of mouse visual acuity. Eur J Neurosci 17:167-173.

Prusky GT, West PW, Douglas RM (2000) Behavioral assessment of visual acuity in mice and rats. Vision Res 40:2201-2209.

Reinert KC, Dunbar RL, Gao W, Chen G, Ebner TJ (2004) Flavoprotein autofluorescence imaging of neuronal activation in the cerebellar cortex in vivo. J Neurophysiol 92:199-211.

Sawtell NB, Frenkel MY, Philpot BD, Nakazawa K, Tonegawa S, Bear MF (2003) NMDA receptor-dependent ocular dominance plasticity in adult visual cortex. Neuron 38:977-985.

Schuett S, Bonhoeffer T, Hubener M (2002) Mapping retinotopic structure in mouse visual cortex with optical imaging. J Neurosci 22:6549-6559. 
Sheth SA, Nemoto M, Guiou M, Walker M, Pouratian N, Toga AW (2004) Linear and nonlinear relationships between neuronal activity, oxygen metabolism, and hemodynamic responses. Neuron 42:347-355.

Shibuki K, Hishida R, Murakami H, Kudoh M, Kawaguchi T, Watanabe M, Watanabe S, Kouuchi T, Tanaka R (2003) Dynamic imaging of somatosensory cortical activities in the rat visualized by flavoprotein autofluorescence. J Physiol (Lond) 549:919-927.

Shibuki K, Hishida R, Kitaura H, Takahashi K, Tohmi M (2006a) Coupling of brain function and metabolism: endogenous flavoprotein fluorescence imaging of neural activities by local changes in energy metabolism. In: Handbook of neurochemistry and molecular neurobiology, Vol V, Neural energy utilization (Gibson G, Dienel G, eds), in press. New York: Plenum.

Shibuki K, Ono K, Hishida R, Kudoh M (2006b) Endogenous fluorescence imaging of somatosensory cortical activities after discrimination learning in rats. NeuroImage 30:735-744.

Shtoyerman E, Arieli A, Slovin H, Vanzetta I, Grinvald A (2000) Long-term optical imaging and spectroscopy reveal mechanisms underlying the intrinsic signal and stability of cortical maps in V1 of behaving monkeys. J Neurosci 20:8111-8121.

Shuttleworth CW, Brennan AM, Connor JA (2003) NAD(P)H fluorescence imaging of postsynaptic neuronal activation in murine hippocampal slices. J Neurosci 23:3196-3208.

Steriade M, Nunez A, Amzica F (1993) A novel slow ( $<1 \mathrm{~Hz}$ ) oscillation of neocortical neurons in vivo: depolarizing and hyperpolarizing components. J Neurosci 13:3252-3265.

Tagawa Y, Kanold PO, Majdan M, Shatz CJ (2005) Multiple periods of functional ocular dominance plasticity in mouse visual cortex. Nat Neurosci $8: 380-388$
Taha S, Hanover JL, Silva AJ, Stryker MP (2002) Autophosphorylation of alphaCaMKII is required for ocular dominance plasticity. Neuron 36:483-491.

Takahashi K, Hishida R, Kubota Y, Kudoh M, Takahashi S, Shibuki K (2006) Transcranial fluorescence imaging of auditory cortical plasticity regulated by acoustic environments in mice. Eur J Neurosci 23:1365-1376.

Takao T, Murakami H, Fukuda M, Kawaguchi T, Kakita A, Takahashi H, Kudoh M, Tanaka R, Shibuki K (2006) Transcranial imaging of audiogenic epileptic foci in the cortex of DBA/2J mice. NeuroReport 17:267-271.

Tohmi M, Takahashi K, Kitaura H, Kudoh M, Shibuki K (2005) Transcranial autofluorescence imaging of cortical plasticity induced by monocular deprivation in the mouse primary visual cortex. Soc Neurosci Abstr 31:979.8.

Wagor E, Mangini NJ, Pearlman AL (1980) Retinotopic organization of striate and extrastriate visual cortex in the mouse. J Comp Neurol 193:187-202.

Weber B, Burger C, Wyss MT, von Schulthess GK, Scheffold F, Buck A (2004) Optical imaging of the spatiotemporal dynamics of cerebral blood flow and oxidative metabolism in the rat barrel cortex. Eur J Neurosci 20:2664-2670.

Wiesel TN, Hubel DH (1965) Extent of recovery from the effects of visual deprivation in kittens. J Neurophysiol 28:1060-1072.

Zhan CA, Ledgeway T, Baker Jr CL (2005) Contrast response in visual cortex: quantitative assessment with intrinsic optical signal imaging and neural firing. NeuroImage 26:330-346.

Zuo Y, Yang G, Kwon E, Gan WB (2005) Long-term sensory deprivation prevents dendritic spine loss in primary somatosensory cortex. Nature 436:261-265. 